\title{
DIDALEX : élaboration d'un outil francophone d'analyse quantitative et qualitative des performances d'élèves du primaire en orthographe lexicale
}

\author{
Florence Mauroux $^{1 *}$, Carole Boudreau ${ }^{2}$, et Marie-France Morin ${ }^{2}$ \\ ${ }^{1}$ Université Toulouse Jean Jaurès, 5 allées Antonio Machado, Toulouse, France \\ ${ }^{2}$ Université de Sherbrooke, 2400, boul. Université, Sherbrooke, Canada
}

\begin{abstract}
Résumé : Depuis plusieurs années, des études menées au Québec et en France révèlent une baisse des performances orthographiques des élèves de niveau primaire. Ces résultats s'appuient souvent sur des systèmes de cotation qui fournissent des données quantitatives mais ne permettent pas une étude qualitative des erreurs produites. Dans le cadre de notre étude, nous avons élaboré un logiciel d'analyse qualitative et quantitative des erreurs d'orthographe lexicale chez de jeunes scripteurs québécois et français, âgés de 6 à 12 ans. En nous inspirant de travaux menés dans différentes langues, nous avons adapté l'outil pour une population francophone. Le système de cotation repose sur la présence des graphies requises, leur utilisation adéquate dans le mot écrit, de même que leur plausibilité lorsque l'élève en fait un usage erroné. Sur le plan scientifique, cette démarche multidimensionnelle d'analyse permet une compréhension plus fine des connaissances graphémiques des élèves dans le domaine de l'écriture. Tant pour le milieu scientifique que celui de la pratique, cette démarche d'analyse permet également de suivre l'évolution des représentations graphémiques des jeunes apprenants à travers le temps et de proposer des interventions scolaires en conséquence. Nous présentons ici le prototype du logiciel DIDALEX.
\end{abstract}

\begin{abstract}
DIDALEX: development of a French-speaking tool of quantitative and qualitative analysis of the performances of students of primary school in lexical spelling

For several years, studies led in Quebec and in France have revealed a reduction of the spelling performances of students of primary school. These results are generally based on systems of quotation that give quantitative data but are not able to document precisely the types of errors made by the students. In our study, we developed a software for a qualitative and quantitative analysis of the errors of lexical spelling of young writers from Quebec and France, from 6 to 12 years old. Based on various works led in different languages, we adapted the approach for a
\end{abstract}

* Corresponding author : florence.mauroux@univ-tlse2.fr 
French-speaking population. The system of quotation is based on the presence of the required written forms, their adequate use in the written word, as well as their admissibility in the student's erroneous use. On the scientific plan, this approach of analysis allows a finer understanding of the graphemic knowledges of the students' writing skills. Both for the scientific circle and the teaching environment, this approach of analysis also allows to follow up the evolution of the graphemic representations of the young learners through time and to propose school interventions accordingly. We present here the prototype of the software DIDALEX.

\section{Introduction}

Dans une société où l'écrit occupe une place de plus en plus importante (Rochex et Crinon, 2011), les enjeux de la maitrise de l'orthographe apparaissent déterminants. Concernant plus particulièrement l'orthographe lexicale, des travaux ont montré son influence, à la fois sur la compréhension en lecture et sur la production textuelle (Graham, Harris et Herbert, 2011). De fait, lorsqu'il écrit, un scripteur qui maitrise l'orthographe lexicale des mots voit sa charge cognitive allégée au profit de compétences d'écriture de plus haut niveau, telle que la planification ou la mise en texte de l'énoncé à produire.

Des études récentes, menées en France (MEN, 2016) et au Québec (MELS, 2012), mettent à jour une baisse du niveau des performances des élèves de l'école primaire en orthographe. Les difficultés semblent plus particulièrement porter sur l'orthographe grammaticale mais, bien que moins fréquentes, les erreurs liées à l'orthographe lexicale sont également en augmentation. Si la recherche des causes de cette baisse au fil des années dépasse le cadre de cette contribution, on peut évoquer des classes plus hétérogènes, des élèves plus jeunes du fait de la suppression progressive du redoublement, un temps scolaire plus réduit dans lequel il est demandé une variété d'enseignements plus importante qu'auparavant (Brissaud et Cogis, 2011). Au-delà de cette baisse de performance, ou plutôt dans le but d'y remédier, il nous semble important de nous intéresser aux progrès des élèves qui continuent de développer leurs compétences en orthographe tout au long de leur cursus scolaire.

Il apparait donc primordial d'évaluer finement les connaissances et compétences des jeunes apprenants, notamment en orthographe lexicale. Or, l'analyse des traces écrites proposée dans les dispositifs d'évaluation à disposition des enseignants repose le plus souvent sur des systèmes de cotation binaires où l'écriture du mot-cible est jugée soit correcte soit erronée. Une étude menée sur une quarantaine de dispositifs d'évaluation proposée en France à l'entrée de l'école primaire (Mauroux et Garcia-Debanc, 2013) montre que ce système de cotation ne renseigne pas sur le type d'erreurs produites, particulièrement lorsque les traces écrites présentent un écart à la norme encore important au tout début de l'apprentissage du lire-écrire. Nous en voyons une illustration récente dans les consignes de cotation des évaluations diagnostiques proposées à l'entrée en première primaire par le Ministère de l'Éducation national français, au début de l'année scolaire 2017/2018. Ainsi, les propositions d'écriture de l'élève aux 4 mots soumis à la dictée (lit, tapis, domino, vélo) peuvent être cotées 1 si «l'écriture du mot est phonétiquement plausible » ou 9 pour toute « autre réponse », 0 étant réservé à l'absence de réponse (MEN, 2017). En l'état, la cotation 9 ne permet pas de distinguer des performances contrastées telles que VO, VLO, HMIAD pour écrire le mot vélo. Elle ne distingue pas les erreurs produites, ni les compétences manifestées dans ces trois productions. De fait, s'il recherche un outil numérique pour parvenir à une analyse qualitative des traces écrites par ses élèves, l'intervenant scolaire sera dirigé vers des correcteurs orthographiques. Or, ces outils 
présentent un intérêt pour étayer l'écriture et la révision orthographique mais ne renseignent pas sur la performance de l'élève en orthographe lexicale.

Ces ensembles de constats questionnent les outils didactiques pour comprendre et valoriser les erreurs des jeunes scripteurs dans ce qu'elles apprennent d'un niveau de conceptualisation de l'orthographe lexicale du français. Afin de répondre à cette préoccupation, notre étude vise à élaborer un logiciel d'analyse des traces écrites des jeunes élèves. L'objectif est tout d'abord scientifique puisqu'il s'agit 1) de documenter les connaissances relatives à l'étude des processus cognitifs et linguistiques manifestés dans les traces produites par des élèves tout au long de la scolarité du primaire et 2) de mieux comprendre les types d'erreurs rencontrées et d'en proposer un suivi dans le temps. Au plan didactique, l'objectif est de fournir aux intervenants scolaires une analyse à la fois quantitative, mais surtout qualitative des traces produites par leurs élèves, permettant d'envisager des démarches d'enseignement en conséquence.

Dans cette contribution, nous présentons le prototype d'un outil Didactique d'analyse des erreurs lexicales (DIDALEX) ${ }^{3}$. Nous exposons dans un premier temps notre démarche méthodologique d'élaboration de l'outil puis les premiers développements et potentialités du logiciel.

\section{Démarche méthodologique}

\subsection{Fondements théoriques de l'outil d'analyse des traces écrites: variables retenues pour l'analyse}

La littérature scientifique relative aux outils numériques permettant une analyse fine des compétences d'orthographe lexicale mobilisées dans les traces écrites des jeunes scripteurs est peu active et fait apparaitre un besoin dans ce domaine.

Dans la vaste étude Lire-Ecrire CP (Goigoux (dir.), 2016) menée de 2013 à 2015 auprès de 2500 élèves, il est proposé un suivi des compétences des élèves du début de $1^{\text {ère }}$ primaire à la fin de $2^{\text {ème }}$ primaire pour évaluer l'influence des pratiques d'enseignement dont ils ont bénéficié durant cette période. Concernant plus particulièrement leurs compétences d'orthographe lexicale, les résultats sont obtenus à l'aide d'un programme qui enregistre les différentes propositions des mots produits par les élèves pour les phrases dictées à 4 moments de l'étude ( 3 en $1^{\text {ère }}$ primaire et 1 en fin de $2^{\text {ème }}$ primaire). A cette fin, le logiciel compile toutes les propositions des élèves qui peuvent relever soit d'erreurs d'orthographe lexicale (anfants* pour enfants), grammaticale (les enfant* pour les enfants) ou les deux (anfan* pour enfants). L'intérêt est que ce programme autorise, entre autres, une étude statistique des propositions observées et met à jour les confusions récurrentes à un moment de l'apprentissage. En l'état, il ne permet cependant pas une analyse fine des erreurs d'orthographe lexicale au sein du mot, des problèmes linguistiques rencontrés par les élèves et des solutions trouvées pour les résoudre.

Dans le domaine du Traitement Automatique du langage (TAL), des outils, plus nombreux, sont utilisés pour analyser le traitement des erreurs en orthographe grammaticale (par exemple, GATE, Cunningham, 2014). A notre connaissance, il en existe peu pour l'orthographe lexicale (voir cependant Nooj05, Silberztein, 2004). Ces outils permettent, dans de larges corpus de textes, d'effectuer des recherches sur certaines catégories grammaticales ou fonctions syntaxiques, ou encore de cerner des phénomènes de morphologie lexicale précis (par exemple, le $\mathrm{T}$ comme morphème lexical). "Cependant, malgré son intérêt évident, le TAL ne s'adapte pas de façon immédiate à l'apprentissage / enseignement des langues et de la linguistique»(Silberztein et Tutin, 2005), ce qui constitue l'un de nos objectifs de recherche. Le transfert de ces outils d'analyse de corpus à 
celle de production d'élèves, souvent non normées et qui nécessite de ce fait un niveau d'analyse linguistique élevé, s'avère très complexe.

Nous avons donc fait le choix de concevoir cet outil pour répondre à notre préoccupation de réaliser des analyses mixtes, quantitatives et qualitatives, portant sur des unités linguistiques de l'ordre du phonème/graphème. Pour cela, nous nous sommes appuyées sur des travaux menés dans différents systèmes d'écriture, en français (Boissière et al., 2007), espagnol (Diaz et Zamudio, 2011) et anglais (Masterson et Apel, 2010).

Toutefois, la transposition en l'état de ces outils au français apparait également difficile et nécessite des aménagements liés à nos préoccupations de recherche. Nous avons notamment identifié certaines limites de l'analyse proposée par l'outil de Boissière et ses collaborateurs (2007). Comme pour les outils en anglais et en espagnol, ces chercheurs s'appuient sur une analyse de traces écrites qui permet une description de la nature de l'erreur, basée sur des critères d'ajout (ex : tornadre*/tornade), omission (checher*/chercher), substitution (fourfir*/fournir) ou déplacement (curvilateur*/cultivateur). Un deuxième niveau d'analyse situe l'erreur au plan phonologique (chase*/chasse), orthographique (le*/lait), morphographique (omission d'accord dans le groupe nominal) ou encore syntaxique (omission de mot). Si nous retenons les critères d'ajout/suppression/substitution/déplacement, nous constatons que l'analyse de chaque mot se fait globalement et ne permet pas de rendre compte des réussites d'encodage de plusieurs unités infra-lexicales. Ainsi, pour checher*/chercher, on ne retient qu'une omission de notation d'un phonème quand 4 sur 5 ont été correctement transcrits. De même, la description de l'erreur apparait pertinente mais elle renseigne ici sur la conséquence de l'erreur et non sur sa cause possible.

Les spécificités du système orthographique du français ${ }^{4}$ justifient également l'adaptation des outils de Masterson et Appel (2010) et de Diaz et Zamudio (2011) qui présentent pourtant les variables d'analyse que nous souhaitons conserver. En appui de ces constats, trois types d'analyse proposée dans ces travaux ont été retenus pour le développement de notre outil:

- $\quad$ La segmentation des mots-cibles en unités linguistiques (Masterson et Apel, 2010 et Diaz et Zamudio, 2011);

- La plausibilité graphémique du choix opéré par le scripteur pour chaque unité linguistique (Masterson et Apel, 2010 et Diaz et Zamudio, 2011) ;

- La description de l'erreur produite par le scripteur pour chaque unité linguistique identifiée (Boissière et al., 2007 ; Masterson et Appel, 2010 ; Diaz et Zamudio, 2011).

\subsection{Configurations techniques de la plateforme DIDALEX}

La plateforme DIDALEX est une application web qui permet la récolte d'informations portant sur des phrases écrites par des élèves du primaire. Les données sont présentées à l'utilisateur sous forme de graphiques et enregistrées sur le serveur dans une base de données MYSQL qui met en relation plusieurs couches de traitement (configuration des phrases, configuration des différentes propositions d'écriture et propositions de l'élève). Ainsi, le logiciel associe tous les segments saisis (production des élèves) à un résultat en se basant sur les tables de correspondance préconfigurées aux différentes couches de traitement. Les données récoltées permettent l'analyse et le suivi de la progression individuelle des scripteurs en apprentissage. Pour ce faire, chaque année, les participants 
devront écrire trois phrases proposées par le logiciel. Les réponses recueillies sont ensuite retranscrites dans le profil de chacun des élèves.

Dans les paragraphes suivants, nous décrivons la configuration des tables de données relatives à la segmentation des phrases dictées, des différentes propositions d'écriture (système de cotation) et au traitement des données élèves saisie dans le logiciel.

\subsubsection{Configuration des phrases : segmentation des mots-cibles en unités linguistiques}

Pour élaborer notre prototype, nous avons procédé à un pré-traitement manuel des motscibles qui consiste à identifier les composantes linguistiques de chacun des mots à écrire. Cette analyse permet d'identifier précisément les unités linguistiques qui semblent acquises ou, au contraire, problématiques pour l'élève. Ainsi, pour chaque mot soumis dans le cadre d'une dictée, les unités graphémiques attendues sont identifiées de manière distinctive. Par exemple, le mot fantôme est découpé en 6 unités linguistiques : f-an-t-ô-m-e.

Ainsi, une unité linguistique peut correspondre à :

- $\quad$ un phonème, transcrit par un graphème, simple (T) ou complexe (AN) ;

- $\quad$ un morphogramme (T de lait; $\mathrm{S}$ de enfants) ;

- un E caduque dont l'absence peut altérer la prononciation du mot (caravan* pour caravane) ;

- $\quad$ une spécificité orthographique du mot ( $\mathrm{S}$ de toujours).

Ce pré-traitement permet le paramétrage des unités graphémiques qui évaluent, en sortie, les productions des élèves pour les différentes unités identifiées du mot-cible, comparées avec la graphie normée et analysées en conséquence, tout en tenant compte de l'enseignement des complexités orthographiques selon les degrés scolaires des élèves. Dans le logiciel, ces données sont enregistrées dans la table de correspondance « Phrases ».

\subsubsection{Configuration des propositions d'écriture : système de cotation}

Notre système de cotation vise à identifier le caractère plausible ou non des choix graphémiques opérés par l'élève au regard de la norme orthographique. Du fait de la polyvalence graphémique qui caractérise le système orthographique du français, un élève peut avoir fait un mauvais choix de graphie pour écrire un mot sans que cela n'altère sa prononciation, ce qui lui confère un caractère plausible au plan phonologique. Par exemple, si l'élève a écrit anfant* pour enfant, le choix graphémique AN en début de mot est plausible bien qu'il constitue une erreur au plan orthographique dans ce contexte.

Notre système de cotation de chaque unité graphémique croise ainsi les 4 opérations d'ajout/omission/ substitution/déplacement (Masterson et Appel, 2010 ; Diaz et Zamudio, 2011 ; Boissière et al., 2007) avec ces critères de plausibilité graphémique :

- $\quad 0$ : absence de proposition (omission de l'unité linguistique ciblée)

- 1: proposition non plausible au plan phonologique (ajout, déplacement ou substitution par un graphème non plausible. Par exemple, A pour AN dans enfat*/enfant)

- 2: proposition plausible au plan phonologique (substitution par un graphème plausible. Par exemple, AN pour EN dans anfant*/enfant);

- $\quad 3$ : proposition normée (EN pour EN dans enfant).

L'échelle de cotation de DIDALEX propose une évaluation graduée allant de l'absence de production à une production de plus en plus normée. La progressivité de l'échelle d'évaluation nous permet de calculer un score pour chaque mot produit par l'élève et d'évaluer sa performance globale pour l'écriture d'une phrase. Ce système de cotation 
permet de réaliser 1) les analyses quantitatives à l'égard des compétences et connaissances orthographiques propres à chacun des élèves ou à un groupe d'élèves et 2) le repérage de phénomènes linguistiques manifestés dans les productions des élèves (par exemple, des confusions sonores).

Ce principe nécessite un paramétrage des propositions correspondant à chaque cotation. Il est réalisé en amont de la saisie des données des élèves. Dans le logiciel, ces données sont enregistrées dans la table de correspondance «Écriture des phrases ».

\subsubsection{Traitement de la production saisie}

Lors de la saisie des données, le logiciel met en relation les tables de données «Phrases » et «Écriture des phrases» et attribue automatiquement la cotation correspondant à la performance de l'élève pour chaque unité linguistique. Pour cela, il est nécessaire de paramétrer, en amont, une liste de productions possibles pour les cotations 2, 3 et 4 . Cette étape vise à envisager la cause des erreurs graphémiques donnant lieu à une cotation 2 (non plausible). Ainsi, au-delà des opérations d'ajout, substitution et déplacement (inversion de lettres), nous avons intégré des catégories relevant de confusions sonores ou visuelles, comme visible sur la figure 1.

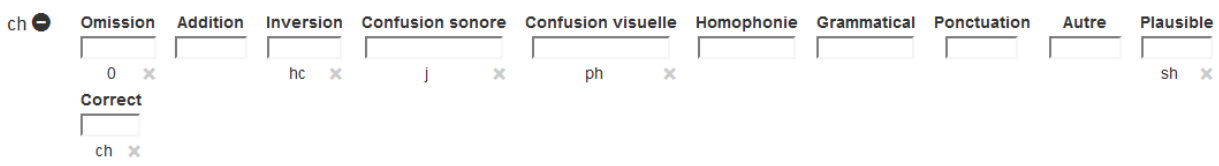

Fig. 1 - Exemple de paramétrage des éléments de saisie pour l'unité linguistique [S] du mot chat

Pour chaque unité linguistique, l'utilisateur a la possibilité de cliquer sur le + , faisant apparaitre les différentes catégories qui peuvent être renseignées. Les propositions saisies seront enregistrées en utilisant le bouton « sauvegarder ».

Les phénomènes d'homophonie étant très fréquents dans le système orthographique du français, nous avons inséré cette catégorie afin d'évaluer le recours à cette procédure pour écrire, sa récurrence, voire son évolution au fil du cursus scolaire. C'est le cas par exemple pour le mot est, dans l'exemple suivant :

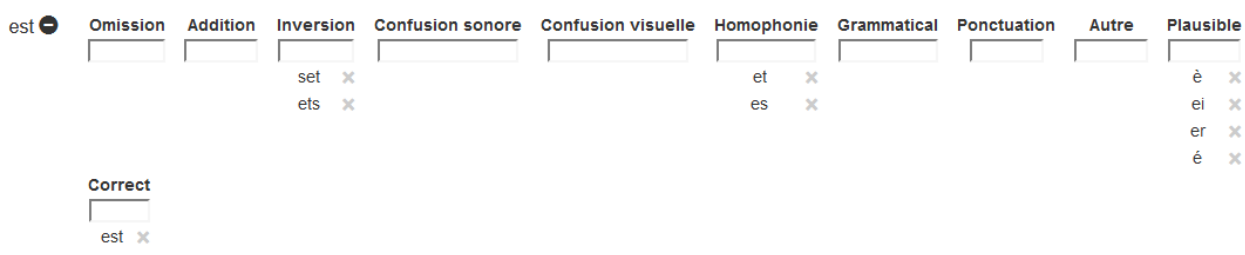

Fig. 2 - Exemple de paramétrage des éléments de saisie pour des mots mobilisant la catégorie « homophonie»

Même si l'objectif premier de notre logiciel est d'évaluer finement l'orthographe lexicale des élèves, nous ne pouvions occulter totalement la dimension d'orthographe grammaticale nécessairement mobilisée dans l'écriture de phrases qui implique un traitement morphosyntaxique. Nous avons donc fait le choix d'insérer une catégorie "grammatical» afin de prendre en compte, à minima, du marquage ou non de la morphologie flexionnelle pour noter l'accord dans les groupes nominaux et les terminaisons des verbes. 
ent $\boldsymbol{\odot}$

Omission Addition Inversion Confusion sonore

Addition
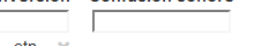

Confusion visuelle Homophonie

eut

Grammatical Ponctuation

Autre

Plausible



(200

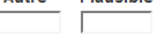

Fig. 3 - Exemple de paramétrage des éléments de saisie pour des mots mobilisant la catégorie champ «Grammatical »

Dans cet exemple, il s'agit de rendre compte des tentatives de l'élève pour noter la marque flexionnelle du verbe dans la phrase «les enfants jouent dans la rue ». Cette catégorie réservée au champ grammatical permet donc d'envisager les choix morphologiques possibles mais non orthographiques.

D'un point de vue plus formel, une catégorie «ponctuation » a également été ajoutée afin de prendre en compte le marquage ou non de cet élément. Si rien n'est saisi dans la case destinée à cet effet, le logiciel conclut à une omission de la marque de ponctuation dans la production de l'élève.

Comme nous le voyons à travers ces illustrations extraites du prototype de DIDALEX, notre outil envisage une description précise des traces produites et des erreurs possibles pour chaque unité linguistique du mot-cible. Au-delà de l'analyse des choix graphémiques de l'élève en comparaison de ceux attendus selon la progression des apprentissages de l'orthographe à l'enseignement primaire, nous espérons ainsi pouvoir repérer des phénomènes linguistiques récurrents, leur moment d'apparition et de résolution au fil du cursus scolaire.

De cette façon, l'outil DIDALEX rempli sa double mission en autorisant à la fois le suivi des progrès de l'élève dans le temps grâce à une analyse qualitative très fine de ses productions et la comparaison des performances d'une cohorte d'élèves possible par le traitement des données quantitatives fournies par les analyses.

Afin de mettre à l'épreuve le prototype de DIDALEX, nous avons constitué une série d'épreuves de dictée de phrases dont nous détaillons à présent l'élaboration.

\section{3 Élaboration d'épreuves d'évaluation des compétences d'orthographe lexicale en primaire}

Pour évaluer les connaissances et compétences orthographiques des élèves tout au long du primaire, nous avons élaboré des épreuves pour chaque degré de l'école primaire. Il s'agit de phrases soumises à dictée en début, milieu et fin d'année scolaire. Le choix des mots constituant les phrases s'est opéré selon deux critères :

- $\quad$ Les unités graphémiques à l'étude pour chaque degré scolaire visé ;

- La fréquence d'utilisation des mots.

Pour cela, les mots ont été sélectionnés à partir des listes orthographiques du ministère de l'éducation du Québec et de la France, en fonction du degré scolaire $\left(1^{\text {ère }}\right.$ à $6^{\text {ème }}$ année primaire au Québec et CP à $6^{\text {ème }}$ année en France). La fréquence des configurations infralinguistiques a également été contrôlée via la plateforme Manulex infra (Peereman, Lété et Sprenger-Charolles, 2007), utilisée tant au Québec qu'en France. Enfin, nous avons considéré le nombre de mots de chaque phrase, leur configuration générale (nombre de 
syllabes du mot) et la variété des types de syllabes qu'ils mobilisent (syllabes de type $\mathrm{V}^{5}$, $\mathrm{VC}, \mathrm{CV}, \mathrm{CVC}, \mathrm{CCV}$, etc.). Ces mots ont été intégrés dans trois phrases de complexité graduée d'un degré scolaire à l'autre. Le tableau 1 présente, pour chaque degré scolaire, les phrases soumises aux élèves en contexte de dictée.

Tableau 1 : Phrases soumises à dictée selon le degré scolaire

\begin{tabular}{|c|c|}
\hline Degré scolaire & Phrases \\
\hline $1^{\text {ere }}-\mathrm{CP}$ & $\begin{array}{ll}1- & \text { Le chat est un animal. } \\
\text { 2- } & \text { Chaque mardi la petite fille va au jardin. } \\
3- & \text { Les enfants jouent avec le ballon jaune dans la rue. }\end{array}$ \\
\hline $2^{\text {ème }}-\mathrm{CE} 1$ & $\begin{array}{ll}1- & \text { Les enfants jouent avec le ballon jaune dans la rue. } \\
\text { 2- } & \text { Pour le carnaval, voudrais-tu te déguiser en fantôme ou en monstre ? } \\
\text { 3- } & \text { Mamie a toujours des idées plein la tête pour occuper mes petits frères. }\end{array}$ \\
\hline $3^{\mathrm{eme}}-\mathrm{CE} 2$ & $\begin{array}{l}\text { 1- Mamie a toujours des idées plein la tête pour occuper mes petits frères. } \\
\text { 2- Cette nuit, je prévois d'apporter une couverture épaisse pour dormir à la } \\
\text { campagne. } \\
\text { 3- Tous les soirs, cet homme observait les étoiles avec sa lunette astronomique. }\end{array}$ \\
\hline $4^{\text {ème }}-$ CM1 & $\begin{array}{l}\text { 1- } \begin{array}{l}\text { Tous les soirs, cet homme observait les étoiles avec sa lunette astronomique. } \\
\text { 2- }\end{array} \text { Par hasard, mon ami a trouvé un livre très ancien dans son grenier. Quelle } \\
\text { découverte incroyable! } \\
\text { 3- } \\
\text { Leur fillette est merveilleuse car elle possède d'immenses qualités et elle est } \\
\text { toujours souriante. }\end{array}$ \\
\hline $5^{\text {ème }}-$ CM2 & $\begin{array}{l}\text { 1- Leur fillette est merveilleuse car elle possède d'immenses qualités et elle est } \\
\text { toujours souriante. } \\
\text { 2- L'artiste est déçu: à la fin de son spectacle il n'a eu que quelques } \\
\text { applaudissements. } \\
\text { 3- Face à une gigantesque tempête, les voyageurs ont dû s'abriter dans un } \\
\text { refuge abandonné. }\end{array}$ \\
\hline $6^{\text {ème }}$ & $\begin{array}{l}\text { 1- Face à une gigantesque tempête, les voyageurs ont dû s'abriter dans un } \\
\text { refuge abandonné. } \\
\text { 2- Cet étudiant est excellent. Il connaît par cœur toutes ses tables de } \\
\text { multiplication. } \\
\text { 3- Fréquemment, des insectes envahissent les champs et créent des ravages } \\
\text { irréparables dans les cultures. }\end{array}$ \\
\hline
\end{tabular}

La dernière phrase dictée en fin d'un niveau scolaire est également celle utilisée en début de niveau scolaire suivant. Ce choix repose sur la volonté d'évaluer les progrès ou, au contraire, la régression, de l'élève après la période des vacances d'été.

La plateforme DIDALEX est configurée pour que chaque utilisateur (intervenant scolaire) puisse saisir les traces écrites par les élèves pour chaque phrase dictée. Nous en présentons l'utilisation dans les paragraphes suivants qui détaillent la première mise à l'épreuve de la plateforme DIDALEX, à partir des épreuves d'évaluation pour la $1^{\text {re }}$ primaire.

\section{Présentation du prototype de la plateforme DIDALEX}

Le but de cette première mise à l'épreuve de la plateforme DIDALEX est de 1) évaluer la fonctionnalité de la plateforme, notamment le processus de saisie des données par un utilisateur lambda, 2) vérifier la pertinence des pré-traitements et paramétrages auxquels s'adossent la saisie des données élèves et 3 ) évaluer les possibilités d'exploitation des 
traces écrites des élèves de primaire via la plateforme DIDALEX. L'objectif général est bien sûr de faire les ajustements nécessaires au regard des constats qui seront posés.

Pour cela, nous disposons de traces écrites par 12 élèves de $1^{\text {ère }}$ primaire, 6 en France et 6 au Québec. Ces élèves ont été soumis à une tâche de dictée de phrase, produite en début (Le chat est un animal.), milieu (Chaque mardi la petite fille va au jardin.) et fin (Les enfants jouent avec le ballon jaune dans la rue.) de l'année scolaire 2016/2017.

\subsection{Accueil de la plateforme DIDALEX}

La procédure de connexion à la plateforme DIDALEX est celle généralement en usage : il suffit de créer un compte avec son adresse courriel à laquelle est associé un mot de passe. 4 onglets sont alors disponibles :

\section{Didalex}

(c) 2018 - CREALEC

Fig. 4 - Extrait de la page d'accueil de la plateforme DIDALEX

- Accueil : La page d'accueil explique les fondements théoriques qui ont présidé à la conception de la plateforme et rappelle ses objectifs didactiques d'aide à l'évaluation et au suivi des compétences des élèves en orthographe lexicale.

- Dictées : c'est à cet emplacement que va se faire la saisie des traces écrites et la cotation automatique. Pour cela, il est nécessaire d'avoir auparavant créé la classe concernée dans l'onglet «mes classes ».

- Mes classes : en cliquant sur l'onglet «Mes classes », on obtient la liste des classes déjà enregistrées (voir fig. 5). Il est alors possible d'en créer une nouvelle en la nommant et en entrant le nom et prénom des élèves qui la composent (fig. 6). 


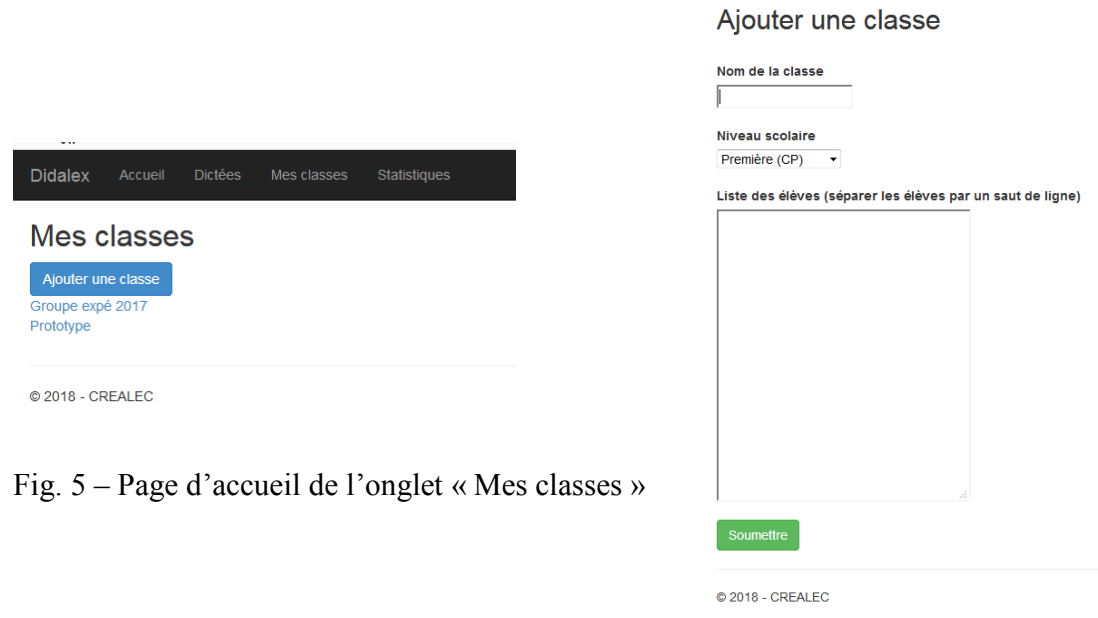

Fig. 6 - Page de création d'une classe

- Statistiques : cet onglet donne accès aux statistiques pour une classe. Nous en détaillons le fonctionnement plus loin.

\subsection{Saisie des traces produites par les élèves}

En arrivant sur l'onglet «Dictée » (Fig. 7), l'utilisateur choisit la classe concernée, la phrase cible et le nom de l'élève dont il veut saisir la production. La page de saisie apparait à l'étape suivante (Fig. 8).

\begin{tabular}{|c|c|c|c|}
\hline Dictées & Phrase: & Élève :? & \multirow{3}{*}{ 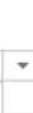 } \\
\hline Classe: & Trimestre 1 & \multirow{6}{*}{$\begin{array}{l}\text { Emilie } \\
\text { Medhi } \\
\text { Maguy } \\
\text { Malone } \\
\text { Johane }\end{array}$} & \\
\hline Prototype & Trimestre 1 & & \\
\hline Groupe expé 2017 & Trimestre 2 & & \\
\hline Prototype & Trimestre 3 & & \\
\hline ○ 2018 - CREALEC & & & \\
\hline & & & \\
\hline
\end{tabular}
trimestre 


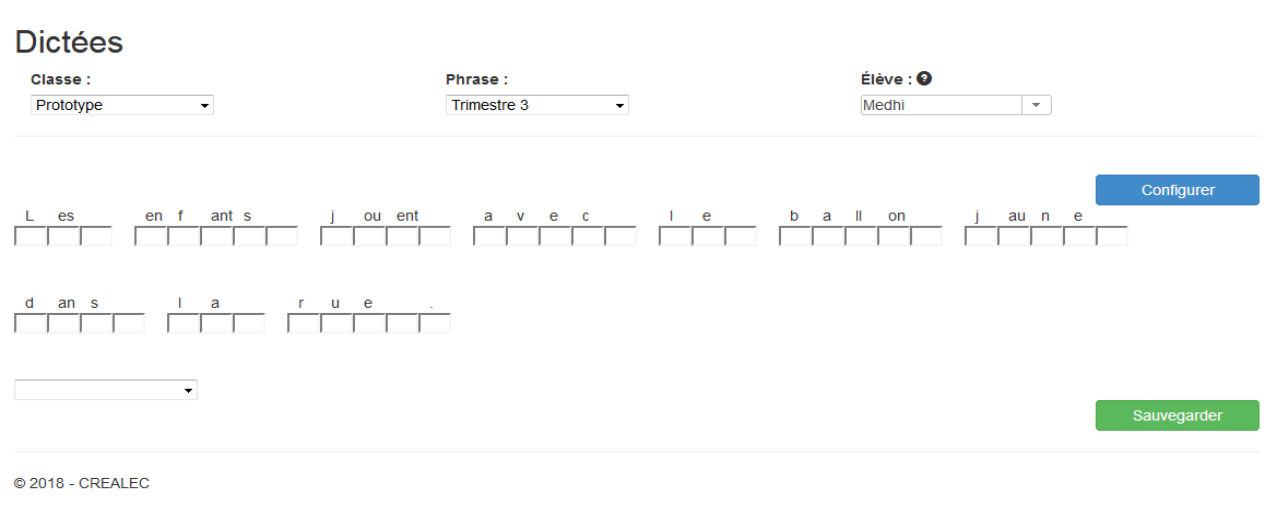

Fig. 8 - Page de saisie des traces produites par l'élève choisi pour $3^{\text {ème }}$ trimestre de $1^{\text {ère }}$ primaire

La phrase dictée apparait, découpée en autant d'unités linguistiques qu'établit lors du prétraitement des mots-cibles. L'utilisateur saisit les unités proposées par l'élève une à une. Il se déplace d'une case à l'autre à l'aide de la touche de tabulation. Au fil de la saisie, le score attribué à chaque élément du mot apparait, comme l'illustre la figure 9.

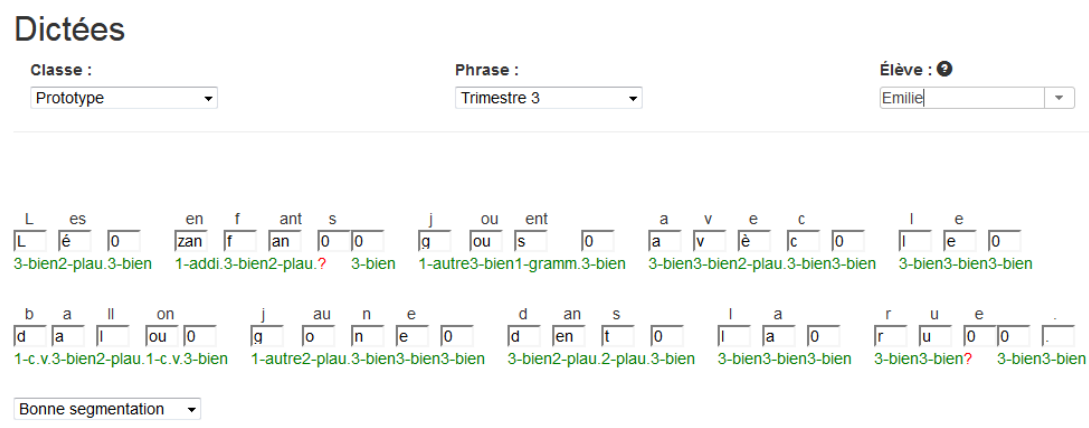

Fig. 9 - Exemple de saisie de la phrase d'Emilie pour le $3^{\text {ème }}$ trimestre de $1^{\text {ère }}$ primaire

Une case laissée vide est automatiquement associée à une non réponse et un point d'interrogation apparait.

Lorsque l'utilisateur saisit une proposition non plausible, le code 1 apparait. Il est suivi d'une abréviation correspondant à la catégorie d'erreurs configurée : inversion de lettres (inv), confusion sonore (c. s.), confusion visuelle (c. v.), homophonie (hom.), grammatical (gram.) ou autre. Cette dernière catégorie est utilisée, par exemple, pour envisager l'utilisation de la valeur épellative d'une lettre ( $\mathrm{K}$ pour écrire la syllabe [ka], ou la méconnaissance de sa valeur contextuelle (par exemple, G pour le phonème [j] dans jouent).

Le code 2-plau s'inscrit lorsqu'une proposition plausible au plan phonétique est faite par l'élève. Cette sortie est possible si le paramétrage a été fait en amont dans la partie « configurer ». Dans le cas contraire, il est possible de configurer une proposition en cours de saisie. 
Pour une proposition normée, le code 3-bien apparait pour une proposition correcte et conforme à celle attendue.

A la fin de la saisie, l'utilisateur évalue la segmentation de la phrase en mots par l'élève, par l'utilisation d'espaces (ou blancs graphiques) entre les mots. Pour cela, il choisit ce qui correspond à la prestation de l'élève dans un tableau déroulant à 4 entrées : aucune segmentation, sous-segmentation, sur-segmentation, bonne segmentation. Cela répond à un constat souvent fait en début d'apprentissage de l'écriture : l'élève peut rencontrer des difficultés à gérer ce paramètre. Il peut alors attacher tous les mots de la phrase (aucune segmentation), seulement quelques-uns (sous-segmentation) ou, au contraire, opérer des séparations au sein des mots (sur-segmentation), le plus souvent au niveau de la syllabe (par exemple : ba lon* pour ballon).

A partir de la saisie de la trace produite par l'élève, l'utilisateur a rapidement accès au score obtenu et à une interprétation possible ayant conduit aux productions erronées. Il procède de même pour tous les élèves de son groupe-classe.

Nous obtenons ainsi un ensemble de données quantitatives et qualitatives. Nous en présentons à présent les exploitations possibles grâce à la plateforme DIDALEX.

\subsection{Exploitation statistique des données}

Cette fonctionnalité de la plateforme DIDALEX est en cours de développement. Notre but est, à terme, de parvenir à un profil de classe à partir des données quantitatives obtenues par la saisie des productions individuelles pour une classe. Ce profil pourrait prendre la forme de celui présenté dans le graphique 1 pour la classe « prototype » :

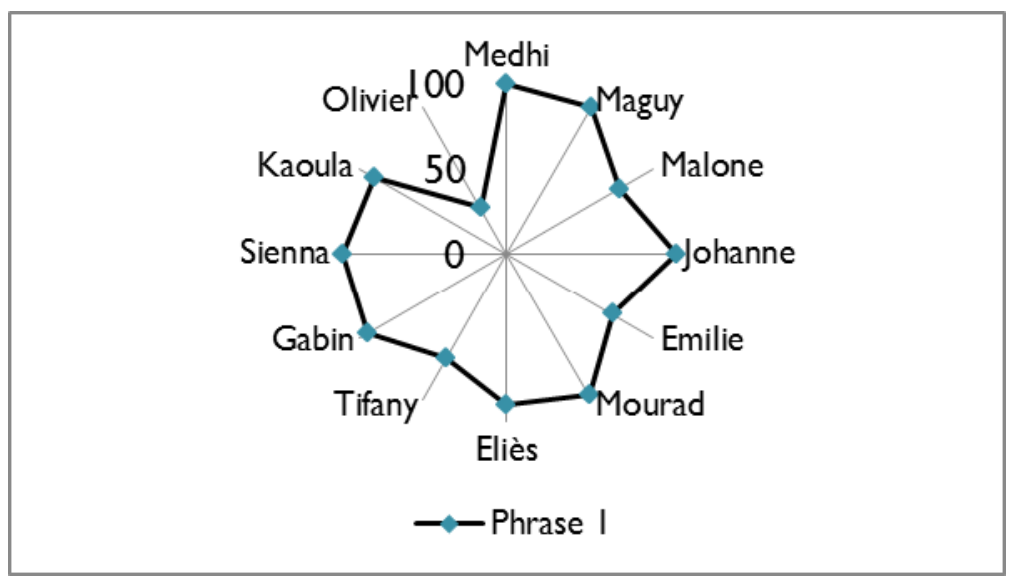

Graphique 1 - Profil du groupe «prototype » à l'écriture de la phrase 1 de 1ère primaire

Le graphique 1 présente, en pourcentages, le score réalisé par chaque élève du groupe à la tâche d'écriture de la première phrase dictée en octobre de $1^{\text {ère }}$ primaire (Le chat est un animal). Dans cet exemple, nous constatons que 7 élèves obtiennent un score proche de $100 \%$. Le graphique permet d'identifier rapidement quatre élèves qui obtiennent une performance inférieure à celle de leurs camarades. Ainsi, Malone, Émilie, Tifany et surtout Olivier semblent rencontrer des difficultés d'encodage. Si ces premières observations ne semblent pas alarmantes pour des élèves de début de $1^{\text {ère }}$ primaire, elles pourront inciter l'intervenant scolaire à une certaine vigilance concernant ces élèves lors des activités d'écriture. 
Afin de suivre l'évolution des élèves dans le temps, la plateforme permettra également d'établir un profil de la classe pour les 3 phrases dictées aux différents moments de l'année scolaire, comme l'illustre le graphique 2 .



Graphique 2 - Profil du «prototype » pour les 3 phrases de $1^{\text {ère }}$ primaire

Ce type de graphique permet de constater que la phrase dictée au $3^{\text {ème }}$ trimestre a globalement posé plus de problèmes aux élèves de ce groupe que les deux phrases précédentes. Le graphique donne à voir certains éléments de progrès aux différents moments de l'année scolaire. Par exemple, Malone obtient un taux de réussite plus élevé pour la phrase 2 , et dans une moindre mesure, pour la phrase 3 que pour la $1^{\text {re }}$ phrase dictée en $1^{\text {re }}$ primaire. A l'inverse, le graphique montre que certains élèves restent en difficulté en écriture. Ainsi, Olivier, Tifany et Kaoula voient leur taux de réussite chuter pour la phrase 2 alors que la majorité des élèves de la classe se maintient à un niveau de performance proche, voire supérieur, de celui obtenu pour l'écriture de la phrase 1.

Ces profils de classe pourront fournir des éléments quantitatifs qui offrent une première cartographie des performances des élèves et permettent de repérer ceux qui nécessitent une vigilance et un accompagnement plus serré de la part de l'enseignant. La taille extrêmement réduite de l'échantillon qui alimente cette perspective de développement ne nous permet pas, en l'état, d'envisager une exploitation statistique plus poussée.

Afin de mieux comprendre les connaissances et compétences mobilisées pour écrire, mais aussi les erreurs produites, la plateforme peut également établir des profils d'élèves s'appuyant sur l'analyse qualitative des données saisies.

\subsection{Exploitation des données qualitatives}

Dans l'onglet «statistiques », l'utilisateur peut avoir accès à un graphique établi à partir des scores obtenus par l'élève à chaque phrase. Nous en voyons une illustration avec le score obtenu par Émilie pour l'écriture de la phrase du $3^{\text {ème }}$ trimestre de $1^{\text {ère }}$ primaire. 


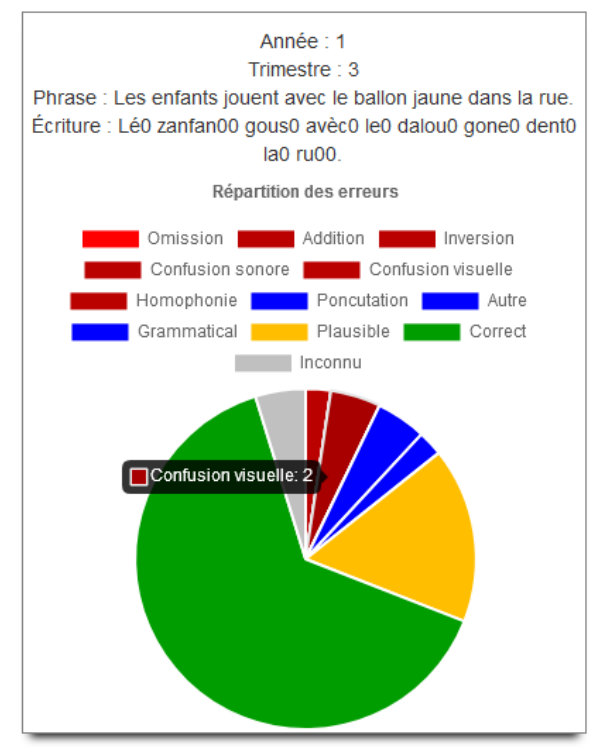

Graphique 3 - Exemple de statistique établis pour un élève pour analyser une phrase

La plateforme calcule automatiquement le taux de réussite de l'élève (proposition correcte) et facilite ainsi l'interprétation des erreurs produites. L'accès à la valeur chiffrée s'obtient en cliquant sur chaque catégorie. Au-delà de la facilité d'utilisation et de l'économie d'analyse liée à l'émission automatique d'interprétation des erreurs, la plateforme permet une recherche par phénomènes linguistiques ou par type d'erreurs.

Ces observations renseignent l'évaluateur à un niveau linguistique très fin. Si elles n'ont rien d'alarmant en première primaire, elles vont orienter l'accompagnement didactique personnalisé de l'intervenant scolaire auprès de l'élève. Les évaluations et analyses ultérieures des productions permettront de mesurer les progrès de l'élève à l'égard des cibles d'interventions mises en place sur le plan de l'orthographe.

\section{Discussion}

Notre premier objectif est d'outiller et de faciliter l'étude des traces écrites produites par les jeunes apprenants. En cela, nous avons pu constater que la plateforme DIDALEX offre un traitement automatisé moins couteux que ne le serait une analyse manuelle pour un ou plusieurs groupes classes.

En proposant une analyse très fine des traces écrites permettant d'inférer les compétences linguistiques qu'elles manifestent, notre deuxième objectif est de mieux comprendre les erreurs produites par les élèves et d'émettre des hypothèses sur leur origine afin de prévoir des actions didactiques au regard des constats posés.

Comme nous l'avons constaté lors de la mise à l'épreuve, la plateforme DIDALEX permet un traitement automatique et rapide des traces saisies pour les élèves d'une classe, et ce malgré un écart à la norme parfois important. L'identification de la nature des erreurs commises par l'élève favorise une interprétation précise de leur origine. En comparaison à des outils à disposition, la plateforme DIDALEX présente un bénéfice important pour l'analyse des traces écrites, notamment sur le plan qualitatif. 
De ce point de vue, il semble que les résultats fournis par la plateforme DIDALEX permettent d'envisager l'erreur non plus comme une faute mais comme la manifestation d'un phénomène linguistique à un moment donné de l'apprentissage. Le suivi dans le temps qu'autorise la plateforme DIDALEX est de nature à renseigner sur les progrès, ou au contraire, l'installation des difficultés rencontrées par un élève. En cela, la plateforme DIDALEX semble remplir sa mission en documentant finement l'analyse compréhensive des traces produites par un élève, ou une cohorte d'élèves, pour accompagner l'enseignement/apprentissage de l'orthographe lexicale.

Ainsi, l'analyse qualitative des productions écrites favorise l'identification des élèves risquant de rencontrer des difficultés dans le développement des compétences en orthographe lexicale. En pointant précisément l'origine et l'ampleur des problèmes manifestés, la plateforme DIDALEX va permettre à l'évaluateur d'envisager des actions didactiques pour faire évoluer les représentations graphémiques des jeunes apprenants et les faire progresser dans la maitrise des compétences ciblées. Au-delà du suivi des progrès, la plateforme DIDALEX pourrait ainsi aider à prévenir l'installation des difficultés en orthographe lexicale. Pour y parvenir, un étalonnage de nos épreuves d'évaluation est prévu auprès d'une cohorte de 1200 élèves de primaire, répartis en France et au Québec, afin de garantir la fiabilité du dispositif.

Pour autant, certains défis méthodologiques demeurent. Le tout premier concerne le corpus de phrases entrées dans l'interface du logiciel, soit 3 phrases par niveau scolaire. En effet, si la saisie des traces produites par les élèves s'est révélée d'un usage aisé pour l'utilisateur, elle ne concerne, actuellement, que les différentes phrases soumises à la dictée pour chaque niveau de l'école primaire. Ce choix s'explique par la complexité du prétraitement que nécessite la segmentation des mots-cibles en unités linguistiques à un grain très fin. Cela concerne également le paramétrage des propositions non plausibles, plausibles et normées, mais aussi des types d'erreurs associées aux propositions non plausibles qui s'opèrent actuellement manuellement. Ces éléments ont motivé notre choix de limiter, dans l'immédiat, le nombre de phrases pré-saisies dans la plateforme. Actuellement, le suivi dans le temps, autorisé par la saisie régulière des traces produites par un élève et/ou une classe, apporte des informations qualitatives déjà nombreuses pour accompagner l'élève dans l'apprentissage et le développement de ses compétences en orthographe lexicale. Nous travaillons cependant à l'automatisation de ces procédures de prétraitement et de paramétrage des propositions, plausibles ou non. En perspective, nous souhaiterions parvenir au développement de la plateforme, permettant la saisie de n'importe quelle trace écrite par un élève dans le cadre d'une production autonome libre.

Un deuxième point de réflexion concerne la catégorisation des erreurs retenue. Comme toute typologie, elle peut en effet prêter à discussion. Ainsi, nous avons souvent dû opérer des choix dans le paramétrage des propositions configurées dans les différentes catégories d'erreur. Par exemple, lorsque le graphème $d$ est attendu dans le mot dans, nous avons choisi de noter $b$ dans les confusions sonores et $p$ et $q$ dans les confusions visuelles. D'autres pourraient sans doute considérer que noter un $b$ pour un $d$ relève également d'une confusion visuelle. Même s'il ne s'agit que d'hypothèses interprétatives, que seul un entretien mené avec l'élève permettrait de valider avec plus de justesse, cette hypothèse est plausible. Il nous a fallu opérer des choix.

Enfin, les choix opérés au plan linguistique sont également appelés à évoluer. En effet, conformément à l'objet d'étude que nous nous sommes fixé, la plateforme DIDALEX permet essentiellement de traiter les erreurs d'orthographe lexicale, le traitement des erreurs d'ordre grammatical n'intervenant qu'à la marge. Cependant, il semble important de pouvoir prendre en compte la gestion faite par l'élève des marques morphographiques, 
nécessairement induites dans l'écriture des phrases proposées à la dictée. En l'état actuel, la catégorie «grammatical» permet de les traiter comme des unités linguistiques à part entière sans que soit clairement identifiée les erreurs commises par l'élève. Par exemple, il serait pertinent de pouvoir distinguer les confusions possibles entre le marquage du nombre au sein du groupe nominal et marquage du verbe, comme la confusion entre les marques flexionnelles S et NT pour les terminaisons verbales (par exemple, ILS JOUS pour ils jouent). Les marques dérivationnelles constituent également un problème à résoudre au plan du traitement automatique. Ces évolutions semblent toutefois importantes pour permettre un suivi dans le temps intégrant la gestion de l'orthographe grammaticale dont la maitrise constitue un défi important pour les apprenants, et ce bien au-delà de l'école primaire (Brissaud et Cogis, 2011).

Pour répondre à ces différentes préoccupations, il est important de rappeler que notre plateforme présente la flexibilité d'une base de données relationnelle qui permet d'envisager une communication avec des logiciels extérieurs et, en perspectives des développements possibles.

\section{Conclusion}

Notre étude a montré la nécessité d'outiller l'analyse des traces écrites des jeunes apprenants pour mieux comprendre comment ils reconstruisent et s'approprient peu à peu les spécificités de l'orthographe lexicale du français. Pour y parvenir, l'appui sur un outil de traitement automatique de saisie des traces écrites de jeunes scripteurs semble un atout important pour accompagner les intervenants scolaires dans cette démarche compréhensive qui accorde un statut positif à l'erreur, témoin de compétences orthographiques en construction. En ce sens, la plateforme DIDALEX semble de nature à répondre aux attentes des institutions, des professionnels chargés de l'enseignement du lire-écrire au primaire mais aussi à celles des didacticiens de l'écriture. La mise à l'épreuve du prototype présenté ici permettra des développements par ajustements successifs aux problèmes soulevés, tant au plan linguistique que technique.

\section{Références bibliographiques}

Boissière, P., Bouraoui, J.-L., Vella, F., Lafarrigue, A., Mojahid, M., Vigouroux, N., Nespoulous, J.L. (2007). Méthodologie d'annotation des erreurs en production écrite. Principes et résultats préliminaires. TALN 2007, Toulouse

Brissaud C. et Cogis D. (2011). Comment enseigner l'orthographe aujourd'hui ? Paris: Hatier.

Cunningham, H. (2014). Developing Language Processing Components with GATE Version 8. University of Sheffield Department of Computer Science. 17 November 2014.

Daigle, D., Ammar, A. et Montesinos, I. (2013). Compétence orthographique et dysorthographie: rôles des procédures explicites. Rapport de recherche. Etude menée dans le cadre de l'action concertée portant sur l'écriture (2010-2013) présenté au fonds québécois de la recherche sur la société et la culture. Gouvernement du Québec.

Direction de l'Evaluation de la Prospective et la Performance, DEPP (2016). Les performances en orthographe des élèves en fin d'école primaire (1987-2007-2015). Note d'information $\mathrm{n}^{\circ} 28$. Novembre 2016. Ministère de l'Éducation nationale, de l'enseignement supérieur et de la recherche. 
Diaz, C. et Zamudio, C. (2011). Desarrollo de una herramienta infórmatica para el análisis de textos infantiles. XVI Congreso Internacional de la ALFAL - Alcalá 2011. Linguistica aplicada, 32473254 .

Goigoux R. (Dir.) (2016). Etude de l'influence des pratiques d'enseignement de la lecture et de l'écriture sur les premiers apprentissages. Synthèse du rapport de recherche. Institut Français de l'Education. Université de Lyon.

Graham, S., Harris, K.R., et Hebert, M. (2011). Informing writing: The benefits of formative assessment. Alliance for Excellence in Education. Washington, D.C. (Commissioned by the Carnegie Corp. of New York).

Mauroux, F. et Garcia-Debanc, C. (2013). Analyse d'épreuves pour évaluer les compétences de scripteur des élèves à l'entrée de l'école élémentaire. Repères (47), 14900171.

Masterson, J., et Apel, K. (2010). Linking characteristics discovered in spelling assessment to intervention goals and methods. Learning Disabilities Quarterly, 33(3), 185198.

Ministère de l'éducation, du loisir et du sport, MELS (2012). Indicateurs de l'éducation, édition 2012. Gouvernement du Québec.

Ministère de l'éducation nationale (2017). Evaluations diagnostiques Français CP. Livret de l'enseignant.

Peereman R., Lete B. et Sprenger-Charolles, L. (2007). Manulex-infra: Distributional characteristics of grapheme-phoneme mappings, and infralexical and lexical units in child-directed written material. Behavior Research Methods, 39 (3), 593-603.

Perfetti C. (1997). «Psycholinguistique de l'orthographe ». In L. Rieben, M. Fayol \& C. Perfetti, Des orthographes et leur acquisition. Paris : Delachaux \& Niestlé, p. 37-56.

Rochex, J.- Y. et Crinon, J. (dir.) (2011). La construction des inégalités scolaires. Au cœur des pratiques et des dispositifs d'enseignement. Rennes : Presses Universitaires de Rennes, $212 \mathrm{p}$.

Silberztein, M. (2004). NooJ : an oriented object approach". In Royauté, J. \& Silberztein, M. (dir.) INTEX pour la Linguistique et le Traitement Automatique des Langues. Proceedings of the 4th and 5th INTEX workshop, Bordeaux, mai 2001 et Marseille, mai 2002. Besançon : Presses universitaires de Franche-Comté.

Silberztein, M. et Tutin, A, (2005). NooJ, un outil TAL pour l'enseignement des langues. Application pour l'étude de la morphologie lexicale en FLE », Alsic [En ligne], Vol. 8, $\mathrm{n}^{\circ} 2$

\footnotetext{
${ }^{3}$ La création de la plateforme DIDALEX a pu se faire grâce au financement de la chaire CREALEC, université de Sherbrooke, Québec, Canada.

${ }^{4}$ Les 26 lettres de l'alphabet latin ne suffisent pas à transcrire les 36 phonèmes du français et l'apprenant devra connaitre et mémoriser environ 130 graphèmes pour lire et écrire. Cette polyvalence graphémique complexifie plus particulièrement la tâche d'écriture. A cette difficulté s'ajoute la nécessité de mobiliser des connaissances morphogrammiques pour noter les morphogrammes (ex : T de lait, $\mathrm{S}$ de cubes dans des cubes) qui sont, le plus souvent, inaudibles, ou des compétences visuo-orthographiques pour mémoriser l'orthographe spécifique de certains mots
} 
(Perfetti, 1997). Ainsi, les caractéristiques du système orthographique du français expliquent que la moitié seulement des mots de la langue française pourront être transcrits régulièrement à partir des correspondances phonographiques (Daigle, Ammar et Montésinos, 2015).

${ }^{5}$ Afin de faciliter la lecture, nous optons ici pour une abréviation des termes Voyelle (V) et Consonne (C). 